\title{
Die Grenzen des Wettbewerbs. Zur Evaluation der Friedens- und Konfliktforschung (nicht nur) aus universitärer Sicht
}

\author{
Thorsten Bonacker
}

Online publiziert: 22. April 2020

(C) Der/die Autor(en) 2020

Zusammenfassung Der Beitrag reagiert auf Empfehlungen des Wissenschaftsrats zur Weiterentwicklung der Friedens- und Konfliktforschung. Er zeichnet nach, wie sich die Friedens- und Konfliktforschung in einer Zeit der Umstellung von einer planerischen auf eine wettbewerblich orientierte Wissenschaftspolitik an Universitäten verankern konnte. Entscheidend dafür waren neben Erfolgen in der Forschungsund insbesondere in der Verbundforschungsförderung die Einführung von Masterstudiengängen. Inzwischen zeichnen sich allerdings zunehmend auch die Grenzen einer solchen „Verwettbewerblichung“ (Winter 2012) für die Entwicklung eines interdisziplinären Forschungsfeldes ab, die in den Empfehlungen des Wissenschaftsrats zum Ausdruck kommen. Im Anschluss an diese Diagnose werden Vorschläge für neue Formen der institutionellen Zusammenarbeit in der Friedens- und Konfliktforschung formuliert.

Schlüsselwörter Friedens- und Konfliktforschung · Wissenschaftsrat · Wettbewerb · Kooperation · Universität

\footnotetext{
T. Bonacker $(\bowtie)$

Zentrum für Konfliktforschung, Philipps-Universität Marburg, Ketzerbach 11, 35032 Marburg, Deutschland

E-Mail: thorsten.bonacker@uni-marburg.de
} 


\title{
The limits of competition. On the evaluation of peace and conflict studies (not only) from a university's perspective
}

\begin{abstract}
This article responds to recommendations of the German Science Council on the further development of Peace and Conflict Studies. It traces how the field of peace and conflict studies was able to anchor itself at universities during the transition from a planning-based to a competition-oriented science policy. In addition to successes in research funding, the introduction of master degree programs was decisive for this success. In the meantime, however, the limits of such a "competition orientation" (Winter 2012) for the development of an interdisciplinary research field are becoming increasingly apparent and are reflected, in the recommendations of the Science Council. Following this diagnosis, proposals for new forms of institutional cooperation in peace and conflict studies are formulated.
\end{abstract}

Keywords Peace and Conflict Studies · Scientific council · Competition · Collaboration · University

\section{Einleitung}

Der Bericht des Wissenschaftsrats hat der Friedens- und Konfliktforschung in Deutschland ein positives Zeugnis ausgestellt und zugleich betont, dass es für die volle Ausschöpfung ihrer Potenziale einer stärkeren Unterstützung durch die Politik bedarf. Die Beiträge in diesem Forum greifen verschiedene Aspekte des Berichts auf, betonen aber dabei fast durchweg die Notwendigkeit von mehr Kooperation innerhalb des Feldes. Beides - die positive Entwicklung und der neue Ruf nach mehr Kooperation - hängt, wie ich im Folgenden zeigen möchte, mit wissenschaftspolitischen Entwicklungen der letzten drei Jahrzehnte zusammen. Mir geht es in meinem Beitrag weniger darum, die Diagnose des Wissenschaftsrats und die damit verbundenen Empfehlungen zu kommentieren. Vielmehr möchte ich den Bericht wissenschaftspolitisch einordnen und dabei zugleich den Prozess der Etablierung der Friedens- und Konfliktforschung an deutschen Universitäten in den Blick nehmen. Dies soll auch dazu beitragen, ein besseres Verständnis dafür zu gewinnen, wie und warum die Friedens- und Konfliktforschung so erfolgreich sein konnte und warum wir nun an einem Punkt angelangt sind, an dem sich die bisherigen Spielregeln der Wissenschaftspolitik wenigstens zum Teil als hinderlich erweisen.

Jahrzehntelang lag der Schwerpunkt der institutionellen Verankerung der Friedens- und Konfliktforschung in Deutschland auf außeruniversitären Institutionen, deren Gründung auf staatliche Entscheidungen zurückging. Zentral dafür waren die Antrittsrede von Bundespräsident Gustav Heinemann 1969 und die im Jahr darauf folgenden Empfehlungen des Wissenschaftsrats (Wissenschaftsrat 1970). Aus ihr ging schließlich eine erste Welle von institutionellen Gründungen hervor. Wie ich im Folgenden argumentieren möchte, ist es ist kein Zufall, dass die universitäre Verankerung der Friedens- und Konfliktforschung zu einem Zeitpunkt geschah, als dieses planerische Verständnis von Wissenschaftspolitik durch ein wettbewerbliches ersetzt wurde. Die „Verwettbewerblichung“ (Winter 2012, S. 17) der Wissenschaft 
und insbesondere der Universitäten, die in den 1990er Jahren beträchtlich an Fahrt gewann, hat der Verankerung der Friedens- und Konfliktforschung an Universitäten genützt - oder besser gesagt, die Friedens- und Konfliktforschung hat die neue Wettbewerbsorientierung zu nutzen gewusst, um sich an Universitäten in Deutschland zu etablieren. Wie der Evaluationsbericht des Wissenschaftsrats zu Recht feststellt, ist dies nicht zuletzt das Verdienst der Deutschen Stiftung Friedensforschung (DSF), die ihrerseits wiederum mit den Anfang der 2000er Jahre bereits gut entwickelten typischen wettbewerblichen Instrumenten - etwa der Ausschreibung einer Förderung für die Entwicklung universitärer Studiengänge - die Konkurrenz zwischen Universitäten um die Einwerbung von Drittmitteln zur Einrichtung der Friedensund Konfliktforschung schürte (Wissenschaftsrat 2019, S. 157).

Die These, die ich in diesem Beitrag vertreten möchte, lautet, dass die Friedensund Konfliktforschung die Verwettbewerblichung in den letzten drei Jahrzehnten zwar dazu zu nutzen vermochte, sich institutionell an Universitäten zu verankern, die Grenzen des Wettbewerbs - oder zumindest bestimmter Formen - für die Entwicklung des Forschungsfeldes aber mittlerweile deutlich zutage treten. Der Bericht des Wissenschaftsrats lässt sich vor diesem Hintergrund als Reaktion auf diese Grenzen des Wettbewerbs verstehen. Er fordert dazu auf, ergänzend zum Wettbewerb vor allem kooperative Elemente zu stärken, um das Potenzial der Friedens- und Konfliktforschung voll zu entfalten.

\section{Die Verwettbewerblichung universitärer Wissenschaft}

War die Organisation von Wissenschaft lange Zeit von politischen Planungsprozessen gekennzeichnet, so änderte sich dies - wenn auch zunächst langsam - zu Beginn der 1980er Jahre. Typische Elemente der Planungsorientierung waren beispielsweise die Kameralistik, aber auch die ministerielle Entscheidung über die Einrichtung von Studiengängen, die eher politisch-administrativen Erwägungen folgte. Die Regierung Kohl hatte Anfang der 1980er Jahre unter dem Motto „Wettbewerb statt Bürokratie" neue Akzente in der Wissenschaftspolitik gesetzt, die schnell Früchte trugen und sowohl vom Wissenschaftsrat als auch von der westdeutschen Rektorenkonferenz positiv aufgegriffen wurden. Die damit vollzogene Einführung von Wettbewerb äußerte sich in der zunehmend projektförmigen Organisation von Forschung ebenso wie in der damit verbundenen sukzessiven Erhöhung des Anteils von Drittmitteln an den Hochschulbudgets (Mayer 2019).

Allerdings beschleunigte sich die Verwettbewerblichung erst ab Mitte der 1990er Jahre mit der Einführung der Hochschulautonomie und des New Public Management für die Hochschulsteuerung. Das neue, heute längst gängige Steuerungsmodell basierte auf der Stärkung von Kompetenzen der Hochschulleitungen bei gleichzeitiger Beschneidung der Entscheidungsbefugnisse von Gremien der Gruppenuniversität, der Einführung von Globalbudgets in den Hochschulen sowie auf Wettbewerb beruhenden bzw. Anreize setzenden Mechanismen der Mittelvergabe. Letztere hingen oft mit der Drittmittelquote einer Universität, aber auch mit den Studierendenzahlen zusammen, deren Steigerung eine zusätzliche Wettbewerbskomponente einführte - 
zumal dann, wenn, wie etwa in Hessen, die Steigerungsquoten der Universitäten relativ zueinander die Grundlage für die Mittelvergabe wurden.

Dieser Wettbewerb blieb keinesfalls nur auf die Landesebene beschränkt, auf der Universitäten um sogenannte ,leistungsorientierte Mittel“" aus den Wissenschaftsministerien konkurrierten - mit der Folge, dass zum einen die vormals für die Mittelvergabe zentralen Stellenpläne an Bedeutung verloren. Zum anderen wurden immer neue spezialisierte Landesprogramme aufgelegt, in denen sich der Wettbewerbsgedanke in der Forschung, aber auch in der Lehre manifestierte - ein Gedanke, der dann später auch auf der Bundesebene mit dem Hochschulpakt oder dem Qualitätspakt Lehre aufgegriffen wurde. Inwiefern es sich um einen echten, marktförmigen Wettbewerb handelt, ist durchaus umstritten. Solange die Hochschulbudgets in weit überwiegendem Maße staatlich finanziert sind, lässt sich, wie Nullmeier (2000) gezeigt hat, eher von einer Intensivierung und Ökonomisierung der Budgetkonkurrenz sprechen als von einem wirklichen Markthandeln, bei dem Universitäten in viel höherem Maße als Anbieter auf Bildungsmärkten auftreten müssen. Stattdessen konkurrieren Universitäten in erster Linie um direkte oder indirekte staatliche Zuwendungen. Selbst die Konkurrenz um Studierende folgt im Wesentlichen finanziellen Anreizen, weil sich die Hochschulbudgets zu einem hohen, wenngleich landesspezifisch unterschiedlich hohen Anteil aus der Zahl der Studierenden ergeben. Daher resümiert Nullmeier: „Vorrangig wird die Situation der Hochschulen heute vom Wettbewerb um öffentliche Finanzmittel beherrscht. Es handelt sich dabei keineswegs um einen Markt, da auf der Anbieterseite (auf Seiten der Länder, des Bundes und der Forschungsförderungseinrichtungen) kein Wettbewerb, sondern ein Monopol (bei den Gebietskörperschaften) bzw. ein Oligopol (bei den Forschungsfördereinrichtungen) herrscht. Auf Seiten der ,Nachfrager' befinden sich dagegen die Hochschulen, die selbst in kleineren Bundesländern in eine Wettbewerbssituation untereinander geraten“ (Nullmeier 2000, S. 217-218).

Die Exzellenzinitiative hat daran nichts Grundlegendes geändert, wenngleich es im Gegensatz zum Wettbewerbsgedanken in den 1990er und frühen 2000er Jahren noch nicht um eine vertikale Differenzierung von Universitäten ging - ein zentrales Ziel der Exzellenzinitiative. Allerdings hat sie den Wettbewerbsgedanken gleichsam in die DNA der Universitäten eingepflanzt, sodass sich auch inneruniversitäre Strategien der Reorganisation zunehmend an ihr ausrichten.

Mitte der 2000er Jahre hatte die Bologna-Reform den Wettbewerb zwischen Universitäten bereits in wenigstens zweifacher Weise erweitert: Erstens hat die Einführung von Bachelor- und Masterstudiengängen institutionelle Konkurrenzen auf einen europäischen Bildungsraum ausgedehnt. Zweitens sehen sich Universitäten in Bezug auf Bildungsabschlüsse zunehmend einer Konkurrenz mit anderen Bildungseinrichtungen wie Fachhochschulen oder Berufsakademien gegenüber, sodass in dieser Hinsicht sogar von einer Entwicklung hin zur Bildung von Märkten gesprochen werden kann, auf denen die Produkte unterschiedlicher Bildungsproduzenten gehandelt werden. Letzteres erhöht den Wettbewerbsdruck auf Universitäten (Krücken 2006).

Die Bologna-Reform hat vor allem dazu geführt, dass die Einrichtung von Studiengängen nicht mehr planerischen Vorgaben folgte, sondern vielmehr auch unter dem Aspekt der Profilierung der Universität betrieben wurde - ein Gedanke, der einer planungsorientierten Wissenschaftspolitik weitestgehend fremd war. Der zu 
erwartende Rückgang der Studierendenzahlen wird zudem die Konkurrenz um Studierende, gerade auch unter den Bedingungen eines transnationalen Bildungsraums, erhöhen. Ablesbar ist dies bereits jetzt an der Einführung englischsprachiger Studiengänge, ebenso wie an dem Aufbau von Marketingabteilungen an den Universitäten. Dies - wie auch andere Elemente der Verwettbewerblichung - sollte allerdings nicht mit einer grundsätzlichen Deregulierung der Wissenschaft verwechselt werden. Vielmehr veranstaltet der Staat „Wettbewerbsverfahren, er schreibt Ziele aus bzw. definiert sie, er bestimmt die Regularien und überwacht deren Einhaltung. Von Staatsferne kann daher keine Rede sein, auch nicht von Deregulierung, sondern vielmehr von Reregulierung“ (Winter 2012, S. 28), die jetzt mit den Instrumenten des Wettbewerbs geschieht.

\section{Der Nutzen des Wettbewerbs für die Friedens- und Konfliktforschung}

Dass sich die Friedens- und Konfliktforschung gerade in der Zeit des Paradigmenwechsels von der planungs- zur wettbewerbspolitischen Steuerung von Hochschulen an Universitäten etabliert hat, ist alles andere als ein Zufall. Ein wichtiger Grund dafür liegt in der Entpolitisierung der Wissenschaftspolitik. Wie der Wissenschaftsrat in seinem Bericht zu Recht feststellt, wies die Friedens- und Konfliktforschung von Anfang an eine Nähe zur Politik auf. Dies galt sowohl für die Nähe zu staatlichen Institutionen - erinnert sei an die vorwiegend direkte staatliche Förderung der Friedens- und Konfliktforschung in den 1970er Jahren - als auch für die Nähe zur Zivilgesellschaft, insbesondere für eine mehr oder weniger enge, allerdings nie spannungsfreie Verbindung zur Friedensbewegung. Friedens- und Konfliktforschung war somit von Beginn an, auf die eine oder andere Art, mit politischen Fragen und öffentlichen Konflikten - von der Wiederbewaffnung in den 1950ern über die atomare Aufrüstung in den 1980er bis hin zur militärischen Intervention in den 1990er Jahren - verwoben. Das macht die Frage nach einer universitären institutionellen Verankerung der Friedens- und Konfliktforschung stets zu einem politischen, und damit zu einem unbequemen und tendenziell kontroversen Thema (siehe Gehring und Rubner 2020). Nicht zufällig war die Förderung der Friedens- und Konfliktforschung aus Bundesmitteln am Ende der Ära Kohl auf einem Tiefststand (Nieth 2016).

Eine demgegenüber stärker am Wettbewerb orientierte Hochschul- und Wissenschaftspolitik folgte anderen als primär politisch-administrativen Kriterien. Es standen Profilierungs- und damit Differenzierungsabsichten, eine generelle Leistungssteigerung und eine Erhöhung der Effizienz im Vordergrund - weitgehend unabhängig von Inhalten und weniger geprägt von realen oder imaginierten gesellschaftlichen Bedarfen. Vor diesem Hintergrund erschien, so meine Vermutung, die Friedens- und Konfliktforschung als eine attraktive Option bei der Entwicklung neuer Studiengänge. Zudem zeigte sie sich im wettbewerblichen Ringen um Fördergelder erfolgreich. Mit anderen Worten, sie nutzte die sich öffnenden Spielräume der Umstellung auf eine wettbewerbliche Hochschulpolitik für eine stärkere institutionelle Verankerung an den Universitäten. 
Die Präsenz der Friedens- und Konfliktforschung an Universitäten beschränkte sich bis Mitte der 1990er Jahre auf einzelne Professuren und Arbeitsgebiete mit einem entsprechenden Schwerpunkt. Im Bericht des Wissenschaftsrats heißt es dazu: „Anders als im außeruniversitären Bereich kam die Institutionalisierung der Friedens- und Konfliktforschung an den deutschen Universitäten in den 1970er Jahren nur zögernd in Gang - am prominentesten waren die beiden 1970/71 eingerichteten Professuren für Innenpolitik und Friedensforschung sowie Internationale Politik und Friedensforschung am Otto-Suhr-Institut der FU Berlin. Darüber hinaus wurden in geringer Anzahl einschlägige Professuren an anderen Universitäten verankert“" (Wissenschaftsrat 2019, S. 18). Stärker fiel demgegenüber eine interdisziplinäre Vernetzung von Wissenschaftlern und Wissenschaftlerinnen an Universitäten ins Gewicht, die sich für friedenspolitische Fragen interessierten. Die daraus resultierende normative Ausrichtung solcher Zusammenschlüsse kann aber zugleich auch als Hinderungsgrund für eine institutionelle Verankerung gesehen werden. Dennoch schufen sie in Universitäten - wie etwa in Marburg, Hagen oder Tübingen - einen fachbereichsübergreifenden Kernbestand interessierter Hochschullehrinnern und -lehrer und damit eine wichtige, vor allem auch personelle Voraussetzung dafür, dass die Möglichkeiten, die sich mit der Veränderung der Wissenschaftspolitik vor allem ab Mitte der 1990er Jahre boten, tatsächlich auch ergriffen werden konnten (Sielschott 2010).

In der zweiten Phase der Verwettbewerblichung der Universitäten spielten vor allem die Studiengangsreformen eine wichtige Rolle. Zunächst hatte die Politik Ende der 1990er Jahre den erneuten Impuls für einen Ausbau und eine darauf folgende Professionalisierung der Friedens- und Konfliktforschung gegeben, indem sie einen Bedarf an wissenschaftlicher Expertise zu den neuen Konfliktdynamiken nach Ende des Ost-West-Konflikts artikulierte. Aber anders als rund 30 Jahre zuvor wurde dieser Bedarf nicht ausschließlich dadurch zu decken versucht, dass man außeruniversitäre Institute gründete. Die mit Mitteln des Bundes als privatrechtliche Stiftung gegründete DSF sollte vielmehr dazu beitragen, diese Expertise zu schaffen - und zwar vor allem auch an den Universitäten. Letzteres wurde an zwei Förderlinien der DSF deutlich, die auf die Stärkung der Friedens- und Konfliktforschung an den Universitäten zielten: die Einrichtung von Masterstudiengängen und die Promotionsförderung. Beide wurden, wie zu diesem Zeitpunkt bereits üblich, in kompetitiven Verfahren vergeben. Dies hatte zwei Konsequenzen: Erstens wurden damit zum ersten Mal die Bedingungen für die Herausbildung eines eigenen wissenschaftlichen Nachwuchses geschaffen, der sich fortan als Friedens- und Konfliktforscher und -forscherinnen verstanden. Für die wissenschaftliche Reproduktion eines Forschungsfeldes zählt dies ohne Frage zu den essenziellen Bedingungen. Zweitens galt Friedens- und Konfliktforschung in den Universitäten, die sich erfolgreich um die Förderung beworben hatten, nunmehr als sichtbarer Teil des institutionellen Alltags der Universität. Dies strahlte offenbar auch auf andere universitäre Standorte ab, die, etwa Frankfurt oder Magdeburg, unabhängig von der Förderung durch die DSF Studiengänge der Friedens- und Konfliktforschung einrichteten. Tanja Brühl spricht in diesem Zusammenhang treffend von der Bologna-Reform als „Türöffner“ und verweist zu Recht darauf, dass die DSF-Förderung auf Profilierungswünsche - aber auch -nöte - der Universitäten traf (Brühl 2015). Die Initiativen zur Ein- 
richtung von Studiengängen der Friedens- und Konfliktforschung befriedigten also gewissermaßen Profilierungs- und Differenzierungsbedürfnisse der Universitäten, die im Zuge der Verwettbewerblichung entstanden waren. Zu beachten ist dabei, dass dies zu sehr unterschiedlichen Organisationsmodellen an den Universitäten geführt hat. Grob gesagt kann hier zwischen zwei Modellen unterschieden werden. Auf der einen Seite wurden Studiengänge in die bestehende Fachbereichsstruktur eingegliedert bzw. als Teil einer Strategie der Studiengangentwicklung von Fachbereichen betrachtet. Der Vorteil dieses Modells besteht zweifellos darin, dass es keiner zusätzlichen und in Universitäten traditionell schwierigen Organisationsreform bedarf. Der Preis besteht allerdings in der vom Wissenschaftsrat beobachteten häufig politikwissenschaftlichen Ausrichtung der Studiengänge, die tendenziell an sozialwissenschaftlichen Fachbereichen angesiedelt wurden. Das zweite, seltenere Modell hat zur Stärkung fachbereichsübergreifender Institute oder Zentren geführt. Friedens- und Konfliktforschung ist hier nicht so sehr als Teil der Politikwissenschaft institutionell verankert, sondern - und darin besteht ein wesentlicher Vorteil - als multi- oder unter Umständen sogar interdisziplinäres Forschungsfeld. Verschiedene Disziplinen partizipieren hier an Studiengängen, wobei es noch eine offene Frage ist, inwiefern sich daraus auch echte interdisziplinäre Kooperationen ergeben. In Bezug auf die Nachhaltigkeit der Verankerung der Friedens- und Konfliktforschung an Universitäten hat dieses Modell den Vorzug, dass es institutionelle Strukturen jenseits einzelner Professuren schafft. Allerdings sind die Hürden dafür angesichts der Trägheit der Universität als Organisation hoch. Die kritischen Hinweise im Bericht des Wissenschaftsrats zu Standorten, an denen die Zukunft der Studiengänge aufgrund von Unterausstattung insgesamt fragil bleibt, zeugen von der Bedeutung einer institutionellen Absicherung und den Vorzügen einer breiteren Beteiligung von Disziplinen und Fachbereichen (Wissenschaftsrat 2019, S. 32).

Mit der Einrichtung von Studiengängen ist es der Friedens- und Konfliktforschung trotz der problematischen Situation an einigen Standorten grundsätzlich gelungen, sich an Universitäten zu etablieren. Dem ging die Gründung des „Arbeitskreises Curriculum" in der Arbeitsgemeinschaft für Friedens- und Konfliktforschung (AFK) voraus, der sich bereits vor der Einrichtung der Studiengänge mit der Frage nach Studieninhalten befasste und der wesentlich von den ersten curricularen Überlegungen zu interdisziplinären Studienangeboten in der Friedens- und Konfliktforschung inspiriert war (Zoll 1996). Nicht zuletzt diesem Umstand - einer der Konkurrenz um Fördermittel und dann um Studierende vorausgehenden gemeinsamen Verständigung über curriculare Inhalte - ist es zu verdanken, dass bis zum heutigen Tage eine intensive, standortübergreifende, gemeinsame Diskussion über die Entwicklung der Studiengänge in der AFK existiert, die seit einigen Jahren auch wichtige Impulse für didaktische Innovationen in der universitären Lehre liefert. Angesichts der berechtigten kritischen Hinweise des Wissenschaftsrats in Bezug auf die teilweise zu knappen Ressourcen der Studiengänge an einigen Standorten, wäre es indes für die Zukunft wünschenswert, wenn sich der Arbeitskreis, aber auch die AFK als solche, noch stärker zum Sprachrohr und zur Vertretung des Forschungsfeldes insgesamt machten, um den Universitätsleitungen inhaltliche und materielle Standards der Lehre überzeugend vermitteln zu können. 
Die jüngste Phase der Verwettbewerblichung hat deutlich gemacht, dass die Konkurrenz um Forschungsgelder - etwa im Rahmen der Exzellenzinitiative - durchaus auch der Friedens- und Konfliktforschung zuträglich für ihre Verankerung an Universitäten war. Das von 2007 bis 2019 im Rahmen der Exzellenzinitiative geförderte Cluster „Normative Orders“ an der Goethe-Universität, aber auch die in der letzten Runde bewilligten Cluster in Konstanz (,Die politische Dimension von Ungleichheit") und Berlin (,Contestations of the Liberal Script") verzeichnen einen substanziellen Anteil der Friedens- und Konfliktforschung. Gleiches gilt für eine Reihe von jüngst abgeschlossenen und laufenden Sonderforschungsbereichen wie etwa die SFBs „Räume begrenzter Staatlichkeit“ an der FU Berlin, „Future Rural Africa“ an der Universität Bonn sowie der SFB/Transregio „Dynamiken der Sicherheit" an den Universitäten in Marburg und Gießen. Ein Grund für diesen Erfolg könnte darin liegen, dass das Forschungsfeld in koordinierten Wettbewerbsverfahren, in denen Interdisziplinarität eine entscheidende Rolle spielt, vielfältige Anschlussmöglichkeiten bietet und für sich genommen in gewisser Weise schon das Signal einer interdisziplinären Ausrichtung sendet. Auch in dieser Hinsicht kommt die Wettbewerbssituation also den Ambitionen des Forschungsfeldes entgegen, sich an Universitäten zu etablieren.

Augenfällig - und vom Wissenschaftsrat nicht unbemerkt geblieben - ist eine gewisse regionale Verdichtung in Hamburg, aber auch in großen Flächenstaaten wie Nordrhein-Westfalen, in denen jeweils mehrere Universitäten und außeruniversitären Institute der Friedens- und Konfliktforschung ko-existieren. In Hessen sind es gleich vier Universitäten mit Professuren oder Zentren - Darmstadt, Frankfurt, Marburg und Gießen. Hinzu kommt mit der Hessischen Stiftung Friedens- und Konfliktforschung (HSFK) das größte außeruniversitäre Institut. Dass dies bislang nicht oder nur im Ansatz zu einer eigenen regionalen Strukturbildung geführt hat, wird vom Wissenschaftsrat ausdrücklich bemängelt, der eine stärkere regionale Vernetzung in seinen Empfehlungen explizit anregt. Die ausbaufähige regionale, aber auch überregionale Verknüpfung universitärer Standorte untereinander sowie mit außeruniversitären Instituten - sieht man vom AK Curriculum in der AFK einmal ab ist dabei, so meine These, gewissermaßen die Schattenseite des Erfolges, den die Friedens- und Konfliktforschung durch die Umstellung auf eine Wettbewerbsorientierung im Hochschulsystem feiern konnte. In ihr manifestieren sich nämlich die Grenzen des politisch induzierten Wettbewerbs zwischen Universitäten, dem nun neue Kooperationsformen an die Seite gestellt werden müssen.

\section{Die Grenzen des Wettbewerbs für die Friedens- und Konfliktforschung}

Zunächst einmal ist grundsätzlich festzuhalten, dass der Wechsel von der Planungszur Wettbewerbsorientierung auch beinhaltet, dass die Hochschulsteuerung nun wesentlich stärker anreizbasiert ist, als dies unter den Bedingungen einer ministeriell verantworteten Hochschulpolitik der Fall war. Das hat zwar zu einer der Friedensund Konfliktforschung in die Karten spielenden Entideologisierung bei der Frage nach der Einrichtung von Studiengängen und der Profilierung von Forschungs- 
schwerpunkten beigetragen. Allerdings bedeutet dies selbstverständlich auch, dass die Friedens- und Konfliktforschung nicht von sich aus einer Universitätsleitung am Herzen liegt und dass der ministerielle Bedarf an wissenschaftlicher Expertise, der für die Entstehung des Forschungsfeldes insgesamt zentral war, für Universitäten zunächst einmal kein Argument darstellt, warum man entsprechende Studienangebote mit dafür notwendigen personellen Ressourcen bereitstellen sollte. Hochschulautonomie bedeutet eben auch, dass sich Universitäten frei von aus ihrer Sicht abstrakten, politischen Erwägungen machen. Anders gesagt hat Verwettbewerblichung für Universitäten eine Diversifizierung von Systemrationalitäten zur Folge, die sie in Betracht ziehen müssen. Hier tritt das politische Beratungsbedürfnis neben andere, ökonomische, wissenschaftliche oder rechtliche Erwägungen. Sichtbar wird dies nicht zuletzt dann, wenn es um die Schließung von Studiengängen oder Instituten geht. Makropolitische Argumente, etwa über die Bedeutung eines Forschungsfeldes für die Bereitstellung von Expertenwissen angesichts zunehmender weltpolitischer Krisen, überzeugen in dieser Diskussion selten, weil sie an ökonomischen oder profilstrategischen Kalkulationen von Universitäten vorbei gehen, die sich selbst wiederum an den regionalen und mittlerweile auch nationalen und transnationalen Wettbewerbsbedingungen orientieren. Dies gilt vor allem dann, wenn Universitäten aufgrund einer strukturellen Unterfinanzierung zwingend darauf angewiesen sind, Fördermittel des Landes oder Bundes einzuwerben. Da die Friedens- und Konfliktforschung für Studierende attraktiv ist und sich mit ihr Studierendenzahlen erhöhen lassen - sie verfügt über ein interdisziplinäres Vernetzungspotenzial, das sich insbesondere für Verbundanträge eignet und da sie zudem aufgrund ihrer inhärenten Anwendungsorientierung leicht einen in der Diskussion um die gesellschaftliche Bedeutung wissenschaftlicher Forschung immer wichtiger werdenden social impact nachweisen kann, bietet sie für die Profilierung von Universitäten viele Potenziale. Zugleich hängt ihre universitäre Verankerung damit in gewisser Weise davon ab, dass Universitätsleitungen diese Potenziale auch als solche wahrnehmen, aufgreifen und mit ihren Strategien beispielsweise bei der Einwerbung von Drittmitteln erfolgreich sind. Wie nachhaltig also die universitäre Verankerung der Friedens- und Konfliktforschung ist, hängt jedenfalls zum Teil von sehr spezifischen Bedingungen und Erwägungen in Universitätsleitungen und -gremien ab. Zudem erwächst daraus für sich genommen eben auch noch keine Kooperation zwischen Standorten.

Gleiches gilt auf regionaler Ebene für das Verhältnis zwischen universitären Standorten. Der mit der Wettbewerbsorientierung verbundene starke Fokus auf Studierendenzahlen mag zwar grundsätzlich der Etablierung der Friedens- und Konfliktforschung entgegengekommen sein, aber er führt auch dazu, dass sich Universitäten als Konkurrenten auf einem - wie auch immer imaginierten - Markt betrachten. Wenn zudem Hochschulpakte durch darin enthaltende Anreize für die Steigerung von Studierendenzahlen oder Landesexellenzförderprogramme Universitäten in ein regionales Wettbewerbsset bringen, dann erschwert das Kooperation in erheblichem Maße.

Davon betroffen ist letztlich bis zu einem gewissen Umfang auch die Entwicklung interdisziplinärer Lehrformate. In Situationen struktureller Unterfinanzierung, in denen knappe Mittel effizient zur Einwerbung weiterer kompetitiver Mittel eingesetzt werden müssen, erscheint die Entwicklung interdisziplinärer Lehrformen leicht 
als zu kostenintensiv. Ein einfaches Beispiel mag dies verdeutlichen: Interdisziplinäre Lehre bindet in der Regel mehr Lehrkapazität als monodisziplinäre - entweder darüber, dass wenigstens zwei Personen eine Lehrveranstaltung durchführen, oder weil sie kapazitär anders berechnet wird. Das wiederum verteuert sie, erst recht, wenn die Natur- und Technikwissenschaften einbezogen werden. Die Empfehlung des Wissenschaftsrats nach einer stärkeren interdisziplinären Vernetzung bedeutet in der Lehre also eine Erhöhung der Ressourcen, von der man Universitäten erst einmal überzeugen muss. Für das Forschungsfeld hingegen ist - will es nicht selbst zu einer Disziplin werden - eine solche interdisziplinäre Vernetzung in der Lehre allein schon deshalb essenziell, weil nur darüber hinreichende Kompetenz für interdisziplinäre Forschung aufgebaut werden kann (Bernshausen und Bonacker 2015). Aus Sicht universitärer Standorte wäre es naheliegend, an dieser Stelle spezielle Förderformate, etwa durch die DSF, für interdisziplinäre Lehre an den Universitäten $\mathrm{zu}$ entwickeln, um der naheliegenden und durch den Wissenschaftsrat kritisierten disziplinären Fokussierung entgegenzutreten.

Neben der Konkurrenz um Studierende verdeutlicht auch die Konkurrenz um Forschungsmittel die Grenzen des Wettbewerbs für die Entwicklung des Forschungsfeldes, weil sie - bislang jedenfalls - nur in begrenztem Maße Kooperation und Abstimmung zwischen Standorten der Friedens- und Konfliktforschung gefördert hat. Die jüngste Ausschreibung des BMBF zur Förderung eines standortverteilten Instituts für gesellschaftlichen Zusammenhalt deutet indes an, dass kooperative Strukturen in Zukunft an Bedeutung gewinnen könnten. Dafür gilt es, sowohl innerhalb des Forschungsfeldes der Friedens- und Konfliktforschung, als auch wissenschaftspolitisch, jetzt die Weichen zu stellen. Aus der Sicht des Forschungsfeldes sollten daher neben der Beteiligung an wettbewerblichen Verfahren in Forschung und Lehre neue Formen der Kooperation entwickelt bzw. die bestehenden institutionellen Möglichkeiten der Zusammenarbeit stärker genutzt werden. Dies gilt umso mehr, als eine Orientierung an wettbewerblichen Verfahren nicht zwingend zur Befriedigung der Interessen des Forschungsfeldes als solchem dient. Zudem müssten diese zuallererst in gemeinsamen Beratungen identifiziert werden. Der Evaluationsprozess hat hier wichtige Impulse gegeben und zu der Erkenntnis geführt, dass es solcher Beratungen und Abstimmungen zunehmend bedarf, um die negativen Effekte einer starken anreizbasierten Steuerung von Wissenschaft abzumildern. Dazu möchte ich abschließend einige Vorschläge unterbreiten.

\section{Für mehr Kooperation innerhalb des Forschungsfeldes}

Wettbewerb gehört zu den wesentlichen Eigenschaften wissenschaftlicher Rationalität. Wer als Zweiter eine Entdeckung macht, wird nicht belohnt. Und wer feststellen muss, dass die eigene Forschungsidee schon andernorts publiziert oder gefördert wurde, wird sich nach einer neuen und originelleren Idee umsehen. Davon zu unterscheiden ist die politische Erzeugung von Wettbewerb in der Wissenschaftspolitik, die Gefahr läuft, Pseudomärkte zu produzieren oder wissenschaftsfremde Rationalitäten über Gebühr in Universitäten zu verankern. Richard Münch und Max Pechmann (2009) haben eindringlich davor gewarnt, dass solche politisch induzierten, 
also wissenschaftsexternen Wettbewerbe den eigentlichen wissenschaftlichen Wettbewerb unterminieren, weil andere wissenschaftliche Kriterien zur Bewertung von Lehre und Forschung herangezogen werden. Obschon ich diese Einschätzung teile und die Norm der Wissenschaftsfreiheit grundsätzlich die Autonomie des wissenschaftlichen Feldes garantieren sollte, ging es mir in diesem Beitrag nicht um die Frage, wieviel Wettbewerb Wissenschaft braucht, sondern einzig darum, inwiefern die Friedens- und Konfliktforschung von der Verwettbewerblichung der Wissenschaft profitiert hat. Es entbehrt nicht einer gewissen Ironie, dass ein Forschungsfeld, das in besonderer Weise - ob zu Recht oder zu Unrecht - in dem Ruf steht, kritisch zu sein, zu den Gewinnern der Umstellung von einer planungs- zu einer wettbewerbsorientierten Wissenschaftspolitik gehört (kritisch dazu Ruf 2019). Wie ich zu zeigen versucht habe, zahlt die Friedens- und Konfliktforschung dafür aber auch einen Preis, der im Bericht des Wissenschaftsrats gewissermaßen beziffert wird. Deshalb kommt er zu Empfehlungen, die zu einem wesentlichen Teil auf die Stärkung der Zusammenarbeit im Forschungsfeld zielen. Dazu gehören das Anstoßen gemeinsamer, standortübergreifender Forschungsvorhaben, einrichtungsübergreifende Formen des Wissenstransfers und eine stärkere interdisziplinäre Kooperation, die vor allem auch Fächer wie die (Sozial-)Psychologie, Pädagogik und Geschichtswissenschaft sowie die Regionalstudien und die Natur- und Technikwissenschaften mit einbezieht, wobei man hier sicherlich auch noch die Geografie und Wirtschaftswissenschaften hinzufügen sollte.

Der Bericht bietet nun die Gelegenheit, die vorhandenen Defizite auszugleichen, die, wie ich zu zeigen versucht habe, wenigstens zum Teil aus der starken Wettbewerbsorientierung resultieren. Die Friedens- und Konfliktforschung sollte die Gelegenheiten, die sich damit eröffnen, nutzen, und zum einen aus sich selbst heraus Kooperation stärken. Zum anderen sollten der vom Wissenschaftsrat adressierten Politik konkrete Umsetzungsangebote der Empfehlungen vorgelegt werden. Dazu möchte ich abschließend vier Vorschläge unterbreiten, die auf diese Empfehlungen für mehr Kooperation reagieren. Sie zielen auf eine weitere Stärkung des Forschungsfeldes jenseits der am Wettbewerb ausgerichteten Handlungsorientierungen, die fraglos weiterhin eine zentrale Bedeutung für den Erfolg und die universitäre Verankerung der Friedens- und Konfliktforschung haben werden.

Erstens ist noch einmal darauf hinzuweisen, dass die interdisziplinäre Zusammenarbeit gerade zwischen unterschiedlichen Wissenschaftskulturen trotz aller wissenschaftspolitischer Rhetorik an Universitäten vor hohen Hürden steht. Solange diese nicht institutionell entsprechend abgesichert oder mit großem Nachdruck innerhalb von Universitäten gefördert werden, bleiben Rufe nach ihrem Ausbau weitgehend fruchtlos. Aus Sicht des Forschungsfeldes scheint es deshalb wichtig, auf institutionalisierte Formen interdisziplinärer Kooperation hinzuwirken, das heißt Friedensund Konfliktforschung auch als interdisziplinäres Forschungsfeld an Universitäten zu verankern. Dies ist bislang, wenn ich es richtig sehe, nur eingeschränkt der Fall. Die Erfahrung hat gezeigt, dass interdisziplinäre Lehre als wichtiger Impulsgeber in Universitäten dienen kann, sodass spezifische niedrigschwellige Förderformate unterstützend wirken könnten. Der Erfolg der frühen DSF-Förderung von Studiengängen und Nachwuchsförderung vedeutlicht aber, dass sich hierüber die Verankerung der Friedens- und Konfliktforschung weiter vorantreiben lässt. 
Zweitens sollte die Friedens- und Konfliktforschung bestehende Institutionen der Vernetzung und Zusammenarbeit noch besser nutzen (siehe Engels und Schetter 2020). Im Evaluationsprozess ist deutlich geworden, dass dies bislang nicht der Fall ist - aus vielen, auch institutionengeschichtlichen Gründen. Der Wissenschaftsrat hat in seinem Bericht hervorgehoben, dass die Friedens- und Konfliktforschung mit der AFK über einen wissenschaftlichen Fachverband verfügt, dem rund 300 Personen angehören. Zugleich verweist er darauf, dass längst nicht alle Wissenschaftlerinnen und Wissenschaftler aus dem Feld der Friedens- und Konfliktforschung Mitglieder der AFK sind. Dies gilt sicherlich auch für andere wissenschaftliche Fachverbände. Nichtsdestotrotz steht und fällt die Autorität eines Fachverbandes, auch gegenüber Hochschulleitungen und anderen wissenschaftspolitischen Akteuren, mit der Zahl seiner Mitglieder und der Partizipation der universitären Standorte und außeruniversitären Institute. Zugleich bietet die AFK mit ihren jährlichen Kolloquien eine ideale Plattform für standortübergreifende Abstimmungen und Initiativen. Dies sollte entsprechend genutzt und institutionalisiert werden - beispielsweise in Form einer ständigen Konferenz der Standorte, die innerhalb der AFK-Kolloquien stattfinden könnte.

Drittens hat der Wissenschaftsrat darauf hingewiesen, dass zwar regionale Verdichtungen der Institutionen der Friedens- und Konfliktforschung entstanden sind, diese aber nur in Ansätzen zu einer eigenen regionalen Strukturbildung geführt haben. Das gilt auch für die universitären Standorte. Für Flächenländer wie Hessen, Nordrhein-Westfalen und Bayern könnte die Einrichtung regionaler Wissensallianzen eine interessante Möglichkeit bieten, verschiedene Standorte der Friedens- und Konfliktforschung - universitäre und außeruniversitäre, aber auch stärker anwendungsbezogene Institutionen wie den Zivilen Friedensdienst oder Akteure aus der kommunalen Konfliktbearbeitung - zusammenzubringen. Hier ist nicht zuletzt die Landespolitik in Abstimmung mit Universitäten gefordert, entsprechende Voraussetzungen zu schaffen, die aber zugleich regionale Forschungsverbünde für wettbewerbliche Verfahren sowohl auf Bundes- als auch auf europäischer Ebene stärken könnten. Darüber hinaus ließen sich auch dadurch neue Impulse für den Wissenstransfer auch jenseits der klassischen Politikberatung schaffen.

Viertens haben die verschiedenen universitären Standorte schließlich - zum Teil in enger Zusammenarbeit mit den außeruniversitären Instituten - in den letzten Jahren auch durch Erfolge in der Forschungsförderung eine steigende Zahl von Promovierenden zu verzeichnen. Dies ließe sich nutzen, um standortübergreifende Promotionsprogramme zu entwickeln, die freilich so konzipiert sein müssten, dass sie nicht nur für das Forschungsfeld, sondern vor allem auch für die Promovierenden einen Mehrwert hätten. Entsprechende Ko-Betreuungen, die verschiedene Expertisen und institutionelle Hintergründe sowie Netzwerke zusammenbrächten, wären eine durchaus vielversprechende Möglichkeit. Dies gälte umso mehr, wenn sich das britische Modell einer Trennung von Betreuung und Begutachtung endlich auch in Deutschland durchsetzen würde - eine Entwicklung, die bereits in Ansätzen an einigen universitären Standorten zu beobachten ist.

Zusammengefasst hat der Bericht des Wissenschaftsrats bereits jetzt wichtige Signale an die Universitätsleitungen und Wissenschaftspolitikerinnen und -politiker gesendet, die es nun aufzugreifen gilt. Ein wichtiger Aspekt dabei ist die Schaffung 
kooperativer Strukturen, um negativen Effekten steigender Konkurrenzen entgegenzuwirken, die selbst wiederum von der Friedens- und Konfliktforschung seit den 1990er Jahren produktiv - unter anderem zur Verankerung des Forschungsfeldes an Universitäten - genutzt wurden. Es geht dabei nicht darum, Wettbewerb durch Kooperation $\mathrm{zu}$ ersetzen, sondern eine gute Balance zwischen beidem $\mathrm{zu}$ finden, durch die die jüngere Erfolgsgeschichte der Friedens- und Konfliktforschung an Universitäten und darüber hinaus fortgeschrieben werden kann.

Funding Open Access funding provided by Projekt DEAL.

Open Access Dieser Artikel wird unter der Creative Commons Namensnennung 4.0 International Lizenz veröffentlicht, welche die Nutzung, Vervielfältigung, Bearbeitung, Verbreitung und Wiedergabe in jeglichem Medium und Format erlaubt, sofern Sie den/die ursprünglichen Autor(en) und die Quelle ordnungsgemäß nennen, einen Link zur Creative Commons Lizenz beifügen und angeben, ob Änderungen vorgenommen wurden.

Die in diesem Artikel enthaltenen Bilder und sonstiges Drittmaterial unterliegen ebenfalls der genannten Creative Commons Lizenz, sofern sich aus der Abbildungslegende nichts anderes ergibt. Sofern das betreffende Material nicht unter der genannten Creative Commons Lizenz steht und die betreffende Handlung nicht nach gesetzlichen Vorschriften erlaubt ist, ist für die oben aufgeführten Weiterverwendungen des Materials die Einwilligung des jeweiligen Rechteinhabers einzuholen.

Weitere Details zur Lizenz entnehmen Sie bitte der Lizenzinformation auf http://creativecommons.org/ licenses/by/4.0/deed.de.

\section{Literatur}

Bernshausen, Sirin, und Thorsten Bonacker. 2015. Ist die Friedens- und Konfliktforschung auf dem Weg zur Disziplin? Ein wissenschaftssoziologischer Blick auf die Bedeutung von Studiengängen für die Ausdifferenzierung von Disziplinen. In Konflikte vermitteln? Lehren und Lernen in der Friedensund Konfliktforschung, Hrsg. Mathias Bös, Lars Schmitt, und Kerstin Zimmer, 247-268. Wiesbaden: Springer.

Brühl, Tanja. 2015. Friedenswissenschaftliche Masterstudiengänge: Nachholende Entwicklung als Erfolgsmodell? In Konflikte vermitteln? Lehren und Lernen in der Friedens- und Konfliktforschung, Hrsg. Mathias Bös, Lars Schmitt, und Kerstin Zimmer, 39-48. Wiesbaden: Springer.

Engels, Bettina, und Conrad Schetter. 2020. Die Gelegenheit nutzen: Die Evaluation der Friedens- und Konfliktforschung durch den Wissenschaftsrat aus der Perspektive der Arbeitsgemeinschaft für Friedens- und Konfliktforschung (AFK). Z Friedens und Konffforsch. https://doi.org/10.1007/s42597020-00027-z.

Gehring, Kai, und Henrik Rubner. 2020. Ein wichtiger Kompass in unruhigen Zeiten. Friedensforschung und die Empfehlungen des Wissenschaftsrates aus Perspektive der Politik. Z Friedens und Konflforsch. https://doi.org/10.1007/s42597-020-00031-3.

Krücken, Georg. 2006. Verschärfte Institutionenkonkurrenz und neue organisationale Ungleichheiten durch Bachelor- und Master-Studiengänge? In Soziale Ungleichheit, kulturelle Unterschiede: Verhandlungen des 32. Kongresses der Deutschen Gesellschaft für Soziologie in München. Teilbd. 1 und 2, Hrsg. Karl-Siegbert Rehberg, 1545-1553. Frankfurt am Main: Campus.

Mayer, Alexander. 2019. Universitäten im Wettbewerb. Deutschland von den 1980er Jahren bis zur Exzellenzinitiative. Stuttgart: Franz Steiner Verlag.

Münch, Richard, und Max Pechmann. 2009. Der Kampf um Sichtbarkeit. Zur Kolonialisierung des wissenschaftsinternen Wettbewerbs durch wissenschaftliche Evaluationsverfahren. In Neue Steuerung von Hochschulen: Eine Zwischenbilanz, Hrsg. Jörg Bogumil, Rolf G. Heinze, 67-92. Berlin: Edition Sigma.

Nieth, Jürgen. 2016. Friedensforschung in der BRD - Entwicklungen, Ergebnisse und Desiderate. In Friedensforschung in Deutschland. Anforderungen an eine „kritische Friedensforschung “, Hrsg. Erhard Crome, 10-38. Berlin: Rosa-Luxemburg-Stiftung. 
Nullmeier, Frank. 2000. „Mehr Wettbewerb!“ Zur Marktkonstitution in der Hochschulpolitik. In Die politische Konstitution von Märkten, Hrsg. Roland Czada, Susanne Lütz, 209-227. Wiesbaden: Westdeutscher Verlag.

Ruf, Werner. 2019. Marktgerechter Frieden. Der Neoliberalismus hat die Friedensforschung entpolitisiert und sie den Gesetzen der Rentabilität unterworfen, Rubikon. https://www.rubikon.news/artikel/ marktgerechter-frieden. Zugegriffen: 2. Jan. 2020.

Sielschott, Stephan. 2010. Friedenswissenschaftliche Lehre im Wandel: Aus der Nische in den Boom? Bedingungen und Bedeutungen der Etablierung friedenswissenschaftlicher Master-Studiengänge. CCS Working Papers, Nr. 12. https://archiv.ub.uni-marburg.de/es/2019/0011/pdf/ccs-wp-12.pdf. Zugegriffen: 27. Dez. 2019.

Winter, Martin. 2012. Wettbewerb im Hochschulwesen. Die Hochschule: Journal für Wissenschaft und Bildung 21(2):17-45.

Wissenschaftsrat. 1970. Empfehlungen des Wissenschaftsrates zur Förderung der Friedens- und Konfliktforschung. Drucksache 1688/70. Berlin: Wissenschaftsrat.

Wissenschaftsrat. 2019. Empfehlungen zur Weiterentwicklung der Friedens- und Konfliktforschung. Drucksache 7827-19. Gießen: Wissenschaftsrat.

Zoll, Ralf. 1996. Friedens- und Konfliktforschung als Studiengang. In Friedens- und Konfliktforschung. Eine Einfiuhrung mit Quellen, Hrsg. Peter Imbusch, Ralf Zoll, 162-193. Opladen: Leske + Budrich. 